\title{
Caracterización del nivel de Aptitud Funcional de Mujeres Mayores Residentes en Comunidades de Bajos Ingresos
}

\author{
Characterizing the level of functional fitness of female senior-citizens \\ residing in low-income communities
}

\author{
Jair Sindra Virtuoso-Júnior ${ }^{1}$ y Ricardo Oliveira-Guerra²
}

1 Departamento de Ciências da Saúde, Universidade Estadual de Santa Cruz. Ilhéus, BA, Brasil. Email: virtuosojr@yahoo.com.br

2 Departamento de Fisioterapia, Universidade Federal do Rio Grande do Norte. Natal, RN, Brasil. E-mail: roguerra@ufrnet.br

Recibido 7 Marzo 2008/Enviado para Modificación 25 Septiembre 2008/Aceptado 15 Octubre 2008

\section{RESUMEN}

Objetivo Desarrollar una tabla descriptiva con el nivel de desempeño en parámetros de aptitud funcional en mujeres de 60 a 92 años, residentes en comunidades de bajos ingresos.

Métodos La muestra se conformó con 222 mujeres de 16 grupos de convivencia para personas mayores en el municipio de Jequié, Bahia (IC=95\%), Brasil. Se analizaron los parámetros de aptitud funcional (fuerza, resistencia aeróbica, flexibilidad, agilidad/equilibrio dinámico y el índice de masa corporal como estimación de la composición corporal), correspondientes a tres grupos etarios. Para el análisis de los datos se utilizaron procedimientos de estadística descriptiva (media, desviación media y distribución de percentiles) y análisis de varianza comparando las tres franjas etarias, categorizadas de acuerdo con los parámetros de aptitud funcional, por intermedio del test de Kruskal Wallis, con $p<0.05$.

Resultados Se observaron diferencias entre los grupos de edad en cuanto a la aptitud funcional, excepto para la composición corporal. Sin embargo, en comparación con otros estudios que enfocaron la temática, se constató que los cambios ocurridos en el desempeño físico, al avanzar la edad cronológica, ocurren de manera más lenta.

Conclusiones Los resultados alcanzados proveen una escala normativa específica a ser utilizada en poblaciones con características similares, la cual puede servir como parámetro para la elaboración de programas más efectivos en el mantenimiento de la aptitud funcional de personas mayores, residentes en comunidades de bajos ingresos.

Palabras Clave: Acondicionamiento físico, factores socioeconómicos, anciano, mujeres (fuente: DeCS, BIREME). 


\section{ABSTRACT}

Objective Developing a descriptive table regarding functional fitness parameters for women aged 62 to 92 residing in low-income communities.

Methods The samples consisted of 222 women divided into 16 senior-citizen social groups in Jequié, Bahia, $(95 \% \mathrm{Cl})$ Brazil. Functional fitness parameters (force, aerobic resistance, flexibility, agility, dynamic equilibrium and body mass index for evaluating body composition) were analysed in women divided into three age-groups. Descriptive statistics (mean, standard deviation and percent distribution) were used for data analysis and analysing variance by comparing the three age-groups classified in accordance with functional fitness parameters (Kruskal Wallis test, $\mathrm{p}<0.05$ ).

Results There was an expressed standard decline in the age-groups' ability classified in relation to the functional fitness parameters being evaluated (except for body composition). However, compared to other studies dealing with the subject, changes occurring in physical performance happened more gradually with advanced age.

Conclusions The results achieved provide a specific normative scale which may be used on populations having similar characteristics in such a way that they can be used as a parameter for preparing more effective programmes for maintaining the functional fitness of senior-citizens living in low-income communities.

Key Words: Physical fitness, socioeconomic factor, senior citizen, woman (source: $\mathrm{MeSH}, \mathrm{NLM})$.

$\mathrm{E}$ l aumento del contingente de personas mayores en el mundo ha despertado el interés de investigadores en diferentes áreas del conocimiento, en la investigación de cuestiones relacionadas con el bienestar humano en su vejez. En el área de la salud, una de las preocupaciones de los gerontólogos es la preservación de la autonomía funcional, o sea, el mantenimiento, en las personas mayores, de la capacidad de ejercer sus actividades cotidianas. El establecimiento de métodos eficaces y seguros en la evaluación de parámetros físicos que indican niveles de comprometimiento de la autonomía funcional pasan a ser esenciales en el diagnóstico de las condiciones de salud en personas mayores.

Algunos parámetros físicos sintetizan las necesidades básicas de este universo de personas en sus actividades de la vida diaria; tales parámetros pueden ser discriminados en los dominios de la fuerza muscular, resistencia aeróbica, flexibilidad, tiempo de reacción, agilidad, equilibrio dinámico y composición corporal (1). 
Como esas características físicas son parámetros que predicen la condición funcional en las personas más viejas, metodologías de evaluación física fueron elaboradas en el medio científico con la intención de asegurar una evaluación segura y eficaz (2,3). Entre las baterías para la evaluación de la aptitud funcional, se destaca la desarrollada por Rikli y Jones (2), por la facilidad de las personas mayores ejecutar los tests propuestos.

El propósito de este estudio fue caracterizar el nivel de aptitud funcional de mujeres de 60 a 92 años, localizadas en una comunidad de bajo nivel socioeconómico. Los levantamientos de tales informaciones posibilitan el desarrollo de normas para la evaluación funcional y la identificación del estatus funcional, siendo útil en el diagnóstico, en el acompañamiento y en la prescripción individualizada de actividades físicas.

\section{MÉTODOS}

Fue realizado un estudio transversal en el municipio de Jequié, localizado a 365 km de Salvador, capital del estado de Bahía, Región Nordeste de Brasil, en el periodo de octubre a diciembre de 2006. La muestra constituida por 222 mujeres mayores con 60 años o más, fue seleccionada de forma probabilística y estratificada por un grupo de convivencia para personas mayores en el municipio. El valor de la muestra fue proporcional al 43,2 \% de la población inicial de 528 ancianos que representa el número total de sujetos participantes en los 16 grupos de convivencia del municipio, estableciéndose un nivel de confianza de un $95 \%$ con un margen de erro de un $5 \%$.

Cuadro 1. Descripción breve de la Batería de Tests adaptada de Fullerton paraevaluación de la aptitud funcional

\begin{tabular}{|c|c|c|}
\hline Parámetro Fisico & Descripoión & Puntuación \\
\hline $\begin{array}{l}\text { Fuerza de Miembros } \\
\text { Inferiores }\end{array}$ & $\begin{array}{l}\text { De pie y con los brazos flexionados junto al torax la } \\
\text { evaluada deberá sentarse y levantarse de la silla en } \\
30 \text { segundos }\end{array}$ & $\begin{array}{l}\text { Númcro de } \\
\text { repeticiones (rep) }\end{array}$ \\
\hline $\begin{array}{l}\text { Fuerza de Miembros } \\
\text { Superiones }\end{array}$ & $\begin{array}{l}\text { La participante sentada en una silla deberá realizar el } \\
\text { meyor número de flexiones posibles en } 30 \text { segundos. } \\
\text { con el brazo dominarte irguendo un peso de } 2 \mathrm{Kg} \text {. }\end{array}$ & $\begin{array}{l}\text { Número de } \\
\text { repeticiones (rep) }\end{array}$ \\
\hline Resistencia aeróbica & $\begin{array}{l}\text { Consiste en la marcha estacioneria en que las } \\
\text { pasadas completas son contabilizadas en el tiempo } \\
\text { de } 2 \text { mirutos. }\end{array}$ & $\begin{array}{l}\text { Número de } \\
\text { pasadas } \\
\text { exmplatas (rep) }\end{array}$ \\
\hline $\begin{array}{l}\text { Flexibilidad del } \\
\text { cuadril }\end{array}$ & $\begin{array}{l}\text { Sentada en una silla, la evaluada debera estender } \\
\text { una pierna y procurer cencucir los bra zos a la meyor } \\
\text { distancia posible en la dirección del Hallux. }\end{array}$ & Centimetro (cm) \\
\hline $\begin{array}{l}\text { Aglidadiequî́brio } \\
\text { dinamico }\end{array}$ & $\begin{array}{l}\text { Estende semtada en una silla, a la seriel de una arden } \\
\text { la evaluada deberá deslocarse para adelante y } \\
\text { contornar un cono colocsdo a una distancia de } 2,4 \text { a } \\
\text { metros con el retorno al puesto inicial del test. }\end{array}$ & Segundos (seg) \\
\hline $\mathrm{MC}$ & Relación del peso con la estatura comporal ( $\mathrm{Kg} / \mathrm{m}^{2}$ ) & $\mathrm{Ka} / \mathrm{mr}^{2}$ \\
\hline
\end{tabular}


Los instrumentos utilizados tuvieron su confiabilidad testada previamente al estudio. Para eso fueron utilizados la Batería de tests físicos de Fullerton (fuerza de miembros inferiores y superiores, resistencia aeróbica, flexibilidad del cuadril, agilidad/equilibrio dinámico e índice de masa corporal) (2)y una entrevista aplicada de forma individual, para la caracterización de la muestra (Tabla 1 y 2) con la evaluación de factores socio-demográficos (edad, educación, clasificación socio-económica) (4) y comportamientos relacionados a la salud (salud percibida, uso de remedios, sintomatología depresiva (5), actividades instrumentales de la vida diaria (6) y hábitos de prácticas de actividades físicas durante el ocio).

Recolección de datos fue realizada por profesionales del área de la salud debidamente entrenados, a través de conferencias y de la aplicación de los instrumentos en el estudio piloto. La entrevista y la aplicación de la batería de tests fueron aplicadas en una sala climatizada y con el piso encauchutado para que las evaluadas pudiesen realizar descalzas los tests propuestos.

Los criterios para la inclusión en el estudio siguieron el modelo de seguridad para la realización de tests físicos en personas mayores (7). Fueron excluidas de los tests las mujeres que presentaron alguna condición médica, limitaciones físicas o cognitivas severas que pudiesen impedir las instrucciones de seguridad para la realización de los tests, siendo que la presión arterial era medida antes y después de la realización de la batería de tests, aquellas que presentaron niveles de presión superiores a 160 mm Hg para la presión arterial sistólica y a 100 mm Hg para la presión arterial diastólica fueron impedidas de realizar tales tests.

Para la confección del banco de datos fue utilizado el software Epidata, versión 3.1b, y los análisis por medio del paquete estadístico SPSS, versión 11. El análisis descriptivo de los datos sirvió para caracterizar la muestra, con la distribución de la frecuencia, cálculo de tendencia central (media, mediana y percentiles) y de dispersión (desvío patrón).

La normalidad de los datos fue verificada por el test de Kolmogorov-Smirnov para las variables analizadas. La ausencia de una distribución normal hizo conque fuese utilizado un test no paramétrico (test de Kruskal-Wallis) para verificar las diferencias de los resultados de los tests de aptitud funcional en relación a la franja etaria $(\mathrm{p}<0,005)$. 
Esta investigación siguió los principios éticos presentes en la Declaración de Helsinki y en la Resolución n 196/96 del Conselho Nacional de Saúde do Brasil. Los protocolos de investigación fueron evaluados y aprobados por el Comitê de Ética em Pesquisa com Seres Humanos da Universidade Estadual do Sudoeste da Bahia.

\section{RESULTADOS}

Las Tablas 1 y 2 muestran un resumen de las características de los participantes de este estudio. Aunque hayan sido observadas algunas variaciones en los grupos etarios establecidos, la población en general, puede ser descripta con una media de apenas 2,8 años $( \pm 3,4)$ de estudio en la enseñanza formal, la mayoría de las participantes presentan baja clasificación socio-económica, siendo que el 72,1 \% ( $n=160)$ se encuentra en las categorías económicas menos favorecidas.

Tabla 1. Características de las participantes (media y desvío patrón)

\begin{tabular}{llccc}
\hline & \multicolumn{4}{c}{ Grupo de Edad (años) } \\
\cline { 2 - 5 } & \multicolumn{1}{c}{$60-69$} & $70-79$ & $80-92$ & $60-92$ \\
\hline $\mathrm{N}$ & 115 & 85 & 22 & 222 \\
Edad (años) & $64,3( \pm 3,3)$ & $74,2( \pm 2,9)$ & $83,6( \pm 4,0)$ & $70,0( \pm 7,2)$ \\
Estatura (cm) & $150,1( \pm 6,6)$ & $148,1( \pm 5,6)$ & $148,4( \pm 8,3)$ & $149,2( \pm 6,5)$ \\
Peso (Kg) & $62,0( \pm 12,8)$ & $58,9( \pm 10,5)$ & $57,9( \pm 13,5)$ & $60,4( \pm 12,1)$ \\
Educación (an̂los) & $2,8( \pm 3,4)$ & $2,4( \pm 3,0)$ & $2,0( \pm 2,0)$ & $2,6( \pm 3,2)$ \\
Cantidad de Remedios & $1,3( \pm 0,4)$ & $1,24( \pm 0,2)$ & $2,0( \pm 0,8)$ & $1,2( \pm 0,4)$ \\
Tiempo libre de Actividad & & & & \\
Fisica (minutos/semana) & $58,3( \pm 104,2)$ & $52( \pm 85,8)$ & $25,9( \pm 36,2)$ & $52,5( \pm 92,5)$ \\
\hline
\end{tabular}

Aunque el 50,9 \% ( $n=113)$ de las entrevistadas haya mencionado que participan de actividades físicas en el ocio, la media del tiempo de práctica en tales actividades fue sólo de 52,5 minutos/semana $( \pm 92,5)$.

Los síntomas depresivos fueron relatados por apenas el 10,4 \% (n=23) de las participantes, pero el uso de remedios fue listado por el 72,2 \% ( $n=167)$, siendo que la media de remedios continuos fue de 1,2 unidades $( \pm 0,4)$. Cabe destacar que el 83,3 \% (n=185) indicó algún tipo de dependencia en la realización de las actividades instrumentales de la vida diaria.

La mediana y los ranks medios de las variables de aptitud funcional de acuerdo con la franja etaria están listados en la tabla 3. El test de KruskalWallis constató diferencias significativas en la mayor parte de los componentes de aptitud funcional evaluados, sólo en lo que se refiere al índice de masa corporal (IMC), que fue utilizado como parámetro indicador de la composición corporal, no fueron encontradas diferencias significativas entre las franjas etarias. 
Tabla 2. Características de las participantes

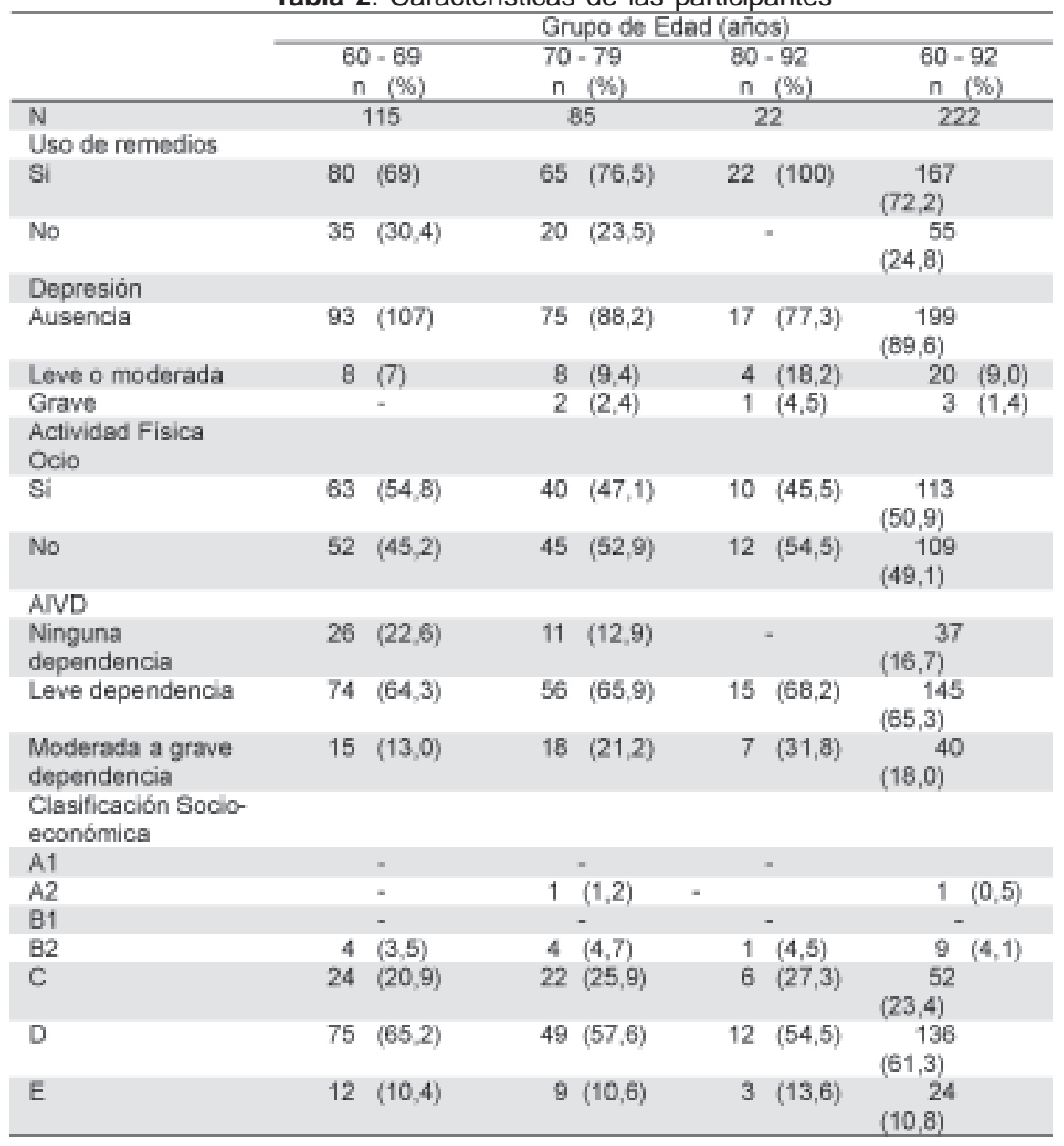

La Tabla 4 contiene las puntuaciones percentiles $\mathrm{P}_{10}, \mathrm{P}_{25}, \mathrm{P}_{50}, \mathrm{P}_{75}$ y $\mathrm{P}_{90}$, equivalente a cada parámetro de aptitud funcional evaluado (fuerza de miembros inferiores y superiores, resistencia aeróbica, flexibilidad, agilidad/equilibrio dinámico y composición corporal) en tres grupos etarios (60-69 años, 70-79 años y 80-92 años).

Los puestos de desempeños descriptos en la Tabla 4 pueden ser evaluados considerando que las puntuaciones que se localicen entre los percentiles $\mathrm{P}_{25} \mathrm{y}$ $\mathrm{P}_{75}$, están distribuidos en la franja considerada de normalidad, o sea, situados próximos a la mediana en una población (2). Los desempeños que media y los cómputos putos por encima del percentil $\mathrm{P}_{75}$, como por encima de la media. Asi, 
en el test de desempeño de la resistencia aeróbica para la edad de 60 a 69 años, el resultado localizado en el intervalo referente a la puntuación de 79 pasadas $\left(\mathrm{P}_{25}\right)$ a la de 106 pasadas $\left(\mathrm{P}_{75}\right)$ sería considerado un resultado normal para aquella población.

Tabla 3. Mediana y Rank medio de los test físicos de las mujeres mayores en

\begin{tabular}{|c|c|c|c|c|c|}
\hline Variables & $\begin{array}{c}\text { Franja etaria } \\
\text { (años) }\end{array}$ & $\mathrm{N}$ & Mediana & Rank Medio & $x^{2}$ \\
\hline $\begin{array}{l}\text { Fuerza de Miembros } \\
\text { Inferiores (número de } \\
\text { repeticiones) }\end{array}$ & $\begin{array}{l}60-69 \\
70-79 \\
80-92\end{array}$ & $\begin{array}{l}123 \\
69 \\
20\end{array}$ & $\begin{array}{l}15,0 \\
14,0 \\
13,0\end{array}$ & $\begin{array}{c}115,72 \\
97,96 \\
79,25\end{array}$ & $\begin{array}{c}8,12 \\
p=0,017\end{array}$ \\
\hline $\begin{array}{l}\text { Fuerza de Miembros } \\
\text { Superiores (número de } \\
\text { repeticiones) }\end{array}$ & $\begin{array}{l}60-69 \\
70-79 \\
80-92\end{array}$ & $\begin{array}{c}124 \\
69 \\
20\end{array}$ & $\begin{array}{l}17,0 \\
15,0 \\
14,0\end{array}$ & $\begin{array}{l}122,07 \\
89,96 \\
72,32\end{array}$ & $\begin{array}{c}19,14 \\
p=0.000\end{array}$ \\
\hline $\begin{array}{l}\text { Resistencia aeróbica } \\
\text { (número de pasos) }\end{array}$ & $\begin{array}{l}60-69 \\
70-79 \\
80-92\end{array}$ & $\begin{array}{l}122 \\
68 \\
20\end{array}$ & $\begin{array}{l}95,5 \\
89,0 \\
74,0\end{array}$ & $\begin{array}{l}115,24 \\
99,49 \\
66,55\end{array}$ & $\begin{array}{c}12,03 \\
p=0,002\end{array}$ \\
\hline Flexibilidad (cm) & $\begin{array}{l}60-69 \\
70-79 \\
80-92\end{array}$ & $\begin{array}{l}126 \\
70 \\
20\end{array}$ & $\begin{array}{l}4,0 \\
2,0 \\
2,0\end{array}$ & $\begin{array}{l}118,65 \\
93,56 \\
96,85\end{array}$ & $\begin{array}{c}8,05 \\
p=0,018\end{array}$ \\
\hline $\begin{array}{l}\text { Agilidad y equilibrio } \\
\text { dinámico (segundos) }\end{array}$ & $\begin{array}{l}60-69 \\
70-79 \\
80-92\end{array}$ & $\begin{array}{l}123 \\
69 \\
20\end{array}$ & $\begin{array}{l}5,37 \\
6,0 \\
6,93\end{array}$ & $\begin{array}{c}85,14 \\
128,14 \\
163,23\end{array}$ & $\begin{array}{c}40,6 \\
p=0,000\end{array}$ \\
\hline IMC $\left(\mathrm{Kg} / \mathrm{m}^{7}\right)$ & $\begin{array}{l}60-69 \\
70-79 \\
80-92\end{array}$ & $\begin{array}{l}129 \\
71 \\
22\end{array}$ & $\begin{array}{l}27,6 \\
26,8 \\
27,1\end{array}$ & $\begin{array}{l}116,24 \\
104,85 \\
105,18\end{array}$ & $\begin{array}{c}1,67 \\
p=0,437\end{array}$ \\
\hline
\end{tabular}

Así el conocimiento de las puntuaciones percentiles para cada parámetro físico puede auxiliar a los profesionales del área de la salud en la detección de los componentes de aptitud funcional que necesitan de perfeccionamiento.

La distribución de los percentiles por grupos etarios se hace necesario, en virtud del declive funcional presentado por los seres humanos después de la fase adulta, siendo que en la vejez hay una aceleración de tales pérdidas funcionales, hecho este que justifica un análisis específico para cada década de vida.

\section{DISCUSIÓN}

La observación más pronunciada de los resultados de este estudio es el patrón relativamente consistente del declive en el desempeño físico de los tests, conforme la variación de la franja etaria. Sin embargo las puntuaciones del índice de masa corporal no presentaron variaciones cuando se los compara con los periodos etarios categorizados en el presente estudio. 
Tabla 4. Grupo de Edad-Percentil

\begin{tabular}{|c|c|c|c|}
\hline \multirow[b]{2}{*}{ Percentil } & \multicolumn{3}{|c|}{ Grupo de Edad } \\
\hline & $60-69$ & $70-79$ & $80-92$ \\
\hline Fuerza de miembros inferiores (reps) & $(n=109)$ & $(n=83)$ & $(n=20)$ \\
\hline $\mathbf{P}_{10}$ & 11.0 & 10,0 & 8.2 \\
\hline$P_{\text {25 }}$ & 13,0 & 11,0 & 11,2 \\
\hline$P_{50}$ & 15,0 & 14,0 & 13,0 \\
\hline$P_{r s}$ & 18,5 & 17,0 & 14,7 \\
\hline$P_{90}$ & 22,0 & 20,0 & 19,8 \\
\hline Fuerza de miembros superiores (reps) & $(n=110)$ & $(n=83)$ & $(n=20)$ \\
\hline$P_{10}$ & 12,1 & 8,4 & 10,1 \\
\hline$P_{25}$ & 14,0 & 13,0 & 11,2 \\
\hline$P_{\infty 0}$ & 17,0 & 15,0 & 14,0 \\
\hline $\mathbf{P}_{76}$ & 20,0 & 18,0 & 15,7 \\
\hline$P_{\infty 0}$ & 22,0 & 20,0 & 17,0 \\
\hline Resistencia aeróbica ( $n^{\circ}$ pasos) & $(n=108)$ & $(n=82)$ & $(n=20)$ \\
\hline$P_{10}$ & 68,9 & 58,0 & 28,5 \\
\hline$P_{25}$ & 79,0 & 72,0 & 59,5 \\
\hline$P_{50}$ & 95,5 & 89,0 & 74,0 \\
\hline$P_{r s}$ & 106,0 & 98,5 & 89,0 \\
\hline$P_{900}$ & 116,4 & 107,7 & 104,9 \\
\hline Flexibilidad (cm) & $(n=112)$ & $(n=84)$ & $(n=20)$ \\
\hline $\mathbf{P}_{10}$ & $-4,0$ & $-7,0$ & $-9,7$ \\
\hline$P_{25}$ & 0,0 & 0,0 & $-0,75$ \\
\hline$P_{50}$ & 4,0 & 2,0 & 2,0 \\
\hline$P_{75}$ & 9,0 & 6,75 & 8,25 \\
\hline$P_{90}^{+}$ & 16,0 & 11,5 & 12,8 \\
\hline Agilidad/Equilibrio (seg) & $(n=109)$ & $(n=83)$ & $(n=20)$ \\
\hline$P_{10}$ & 6,9 & 7,8 & 8,8 \\
\hline$P_{2 s}$ & 5,8 & 7,0 & 7,6 \\
\hline$P_{50}$ & 5,3 & 6,0 & 6,9 \\
\hline$P_{n s}$ & 4,8 & 5,5 & 6.2 \\
\hline$P_{\infty 0}$ & 4,3 & 5,0 & 5,6 \\
\hline IMC $\left(\mathrm{Kg} / \mathrm{m}^{2}\right)$ & $(n=115)$ & $(n=85)$ & $(n=22)$ \\
\hline$P_{10}$ & 21,0 & 21,4 & 17,4 \\
\hline$P_{26}$ & 23,9 & 23,5 & 22,1 \\
\hline$P_{50}$ & 27,6 & 26,8 & 27,1 \\
\hline$P_{r s}$ & 31,1 & 29,1 & 32,3 \\
\hline$P_{\infty}$ & 33,6 & 33,2 & 342 \\
\hline
\end{tabular}

De forma interesante, el declive de los aspectos funcionales observados son consistentes cuando se los compara a publicaciones que se refieren al comportamiento del desempeño físico en tareas motoras cuando se las relaciona con la edad $(8,9)$. Más, las puntuaciones representativas al $\mathrm{P}_{50}$ de los parámetros de fuerza de miembros inferiores y superiores se mostraron diferentes con los indicadores presentados en la literatura para tal asunto.

En general, hay un entendimiento del mayor declive de la fuerza de miembros inferiores que para la fuerza de miembros superiores con el avance de la edad (8). Tal hecho resulta de la disminución del nivel de actividad física en el envejecimiento, principalmente con la reducción de actividades de traslado, 
por otro lado, las actividades domésticas exigen que la parte superior del cuerpo se mantenga más activa y en consecuencia, el declive de la fuerza de miembros superiores pasa a ser menos acentuado cuando se lo compara con la fuerza de miembros inferiores.

Los datos presentados revelan un hecho interesante en lo que se refiere a la baja condición socio-económica del grupo investigado, algo que tal vez lo diferencie de los demás estudios conocidos en la literatura y que fueron producidos con base en muestras de regiones más favorecidas económicamente. En las regiones marginalizadas, en que las personas viven en situaciones precarias económicamente ocurre algo que puede referirse como "contradictorio" para esas personas, por el hecho de que las necesidades laborales son preservadas por largo periodo, y aún, las exigencias en las actividades realizadas exigen esfuerzos vigorosos, a ejemplo de amplios traslados, subida de pendientes y escaleras, y carga de pesos. Por lo tanto, lo contradictorio se da en función de que cuanto más "sufridas" son las condiciones diarias de supervivencia, de una forma mejor esas personas consiguen preservar sus condiciones físicas.

La capacidad aeróbica evaluada por medio del test de marcha estacionaria en el periodo de 2 minutos, apuntó índices esperados de declive de este componente (10). Hay un entendimiento de una variación esperada entre 5 \% y 15 \% por década en relación a las tasas de declive de la capacidad aeróbica $(11,12)$. Los datos analizados a pesar de ratificar que decrece la capacidad aeróbica que es presentada en la literatura mostró por otro lado, que tal declive ocurrió en los límites más bajos de caída esperado, presentando una reducción del 6,8 \% al 14,1 \% respectivamente para la variación de la franja etaria de 60 a 69 años para la de 70 a 79 años y de ésta para la de 80 a 92 años.

A pesar de existir pocos estudios publicados relacionados a los parámetros físicos de flexibilidad, agilidad, equilibrio dinámico relacionados con la edad, algunas publicaciones revelan la disminución de esos componentes con el envejecimiento $(11,13)$. Tal hecho confirma el declive de esos componentes expresados en las Tablas 3 y 4 , sin embargo, el hecho que llama la atención es el acentuado declive del componente flexibilidad (53,6 \%) cuando se compara al grupo de 60 a 69 años en relación a la franja etaria de 80 a 92 años.

Los puntos de corte de IMC para personas mayores todavía son controvertidos debido a los cambios corporales y a las patologías ocurridas en la senilidad, lo que sugiere criterios de clasificación del IMC diferenciados a otras franjas etarias (14). Sin embargo, en el medio especializado se adoptan crite- 
rios de la Organización Mundial de Salud (15) por ser un procedimiento utilizado en estudios en diferentes regiones $(16,17,18)$. El análisis hecho por medio del test Kruskall-Wallis, no indicó una variación significativa de las puntuaciones de IMC en relación a las categorías etarias establecidas en el estudio. Sin embargo, la media de IMC fue de $27,1 \mathrm{Kg} \mathrm{m}^{2}$ caracterizando al grupo de una forma general con sobrepeso, habiendo una amplitud de $16.1 \mathrm{Kg} \cdot \mathrm{m}^{2}$ a $44.9 \mathrm{Kg} . \mathrm{m}^{2}$.

Los datos de la Investigación Nacional sobre Salud y Nutrición realizado hace casi dos décadas, con muestras probabilísticas de las cinco regiones de Brasil y con la participación de 2249 mujeres con edad igual o superior a 60 años, describieron el perfil nutricional de la población mayor brasileña. El estudio identificó prevalencias entre las mujeres mayores entrevistadas de un 8,4 $\%$ de bajo peso (IMC $<18.5 \mathrm{Kg} . \mathrm{m}^{2}$ ), evidenciando un aumento de la frecuencia de bajo peso en la franja etaria de 70 años y un 50,2 \% de sobrepeso (IMC $\geq 25 \mathrm{Kg} . \mathrm{m}^{2}$ ) (18). Tales datos son semejantes a los presentados por otros estudios más recientes, en diferentes regiones del país (19).

El nordeste brasileño, así como otras regiones del país, viene pasando por un fenómeno denominado de transición nutricional, en que el problema de escasez de alimento ha sido sustituida por el exceso de alimentos ricos en grasas, generando así un aumento del sobrepeso y de la obesidad (20). El expresivo aumento del sobrepeso en la población mayor brasileña ya es evidenciado con más intensidad principalmente en las clases económicas intermediarias y bajas $(18,19)$.

Otro hecho a considerar se refiere a que la muestra del presente estudio se constituye sólo de mujeres. Algunos levantamientos han verificado que la prevalencia de sobrepeso/obesidad es mayor en mujeres comparados con hombres adultos ( $<20$ años) (20,21). Los datos presentados por el Instituto Brasileiro de Geografia e Estatística, en lo que respecta a la proporción de personas mayores por género evidencian una expresiva predominancia de las mujeres sobre los hombres en Brasil (22). Siendo que las mujeres mayores cuando se las compara a los hombres de la misma franja etaria y estrato social tienen mayor chance de sufrir de enfermedades crónicas (22).

Como consecuencia de las características socio-demográficas peculiares de los participantes de este estudio (Tablas 1 y 2) se observa que hay diferencias contrastantes en relación a otros estudios poblacionales que describen las condiciones de personas mayores que viven de modo independiente y en condiciones sociales más favorecidas $(8,10,14)$. Las diferencias destacadas no 
son en relación a la tendencia observada del decrecimiento de la aptitud funcional con el envejecimiento. Pero se da en relación a la velocidad de tal declive, en que parece ocurrir más lentamente. Tal hecho sugiere que los resultados normativos de este estudio, sean aplicados en poblaciones con menor nivel económico y educacional.

Conviene resaltar que los procesos de transición demográfica y epidemiológica en América Latina son claramente heterogéneos y están asociados, en gran parte, a las desigualdades y condiciones sociales desfavorables. Los levantamientos de normas de aptitud funcional en poblaciones de personas mayores necesitadas y marginalizadas son escasos existiendo la necesidad de realizar estudios que aborden condiciones de vida y características físicas de personas que viven en regiones menos favorecidas, con la intención de establecer tablas normativas que puedan servir como parámetro de evaluación de las condiciones funcionales de tales grupos.

En conclusión, con respecto a la población investigada, los datos presentados suministran información sobre variaciones normales de los componentes de aptitud funcional en relación a franjas etarias suministrando una escala normativa para una evaluación subsiguiente del individuo y su comparación con el grupo con características similares. Así, la Tabla 4 presentada en ese estudio servirá como una herramienta para el establecimiento de normas de evaluación de poblaciones de baja renta y de menor nivel educacional, con la posibilidad de planeamiento de acciones más efectivas en el control del declive de la aptitud funcional con el avance de la edad cronológica •

\section{REFERENCIAS}

1. Collins KBS, Rooney BL, Smalley KJ, Havens SBS. Functional fitness, disease and independence in community-Dwelling older adults in Western Wisconsin. Wis Med J 2004; 103(1):4248.

2. Rikli RE, Jones CJ. Development and validation of a functional fitness test for communityresiding older adults. J Aging Phys Act 1999; 7:129-61.

3. Cress ME, Buchner DM, Questad KA, Esselman PC, deLateur BJ, Schwartz RS. Continuousscale physical functional performance in healthy older adults: a validation study. Arch Phys Med Rehabil. 1996; 77:1243-50

4. Associação Nacional de Empresas de Pesquisa. Critério de Classificação Econômica Brasil [Internet]. São Paulo. Disponível en: http://www.abep.org/codigosguias/ ABEP_CCEB.pdf. Consultado Febrero 2003.

5. Almeida OP, Almeida AS. Confiabilidade da versão brasileira da escala de depressão em geriatria (GDS) versão reduzida. Arq Neuropsiquiatr 1999; 57(2-B):421-426.

6. Cardoso V, Gonçalves LHT. Instrumentos de avaliação da autonomia no desempenho das atividades da vida diária do cliente idoso. ACM 1995; 24(4):41-48. 
7. Malmberg JJ, Miilunpalo SI, Vuori IM, Pasanen ME. A health-related fitness and functional performance test battery for middle-aged and older adults: feasibility and healthrelated content validity. Arch Phys Med Rehabil 2002; 83:666-677.

8. Newman AB, Haggerty CL, Goodpaster B, Harris T, Kritchevsky S, Nevitt M, Miles TP, Visser M. Strength and muscle quality in a well-functioning cohort of older adults: The health, aging and body composition study. J Am Geriatr Soc 2003; 51(3):323-330.

9. Doherty TJ. Physiology of aging invited review: aging and sarcopenia. J Appl Physiol 2003; 95: 1717-1727.

10. Rikli RE, Jones CJ. Functional Fitness Normative Scores for Community-Residing Older Adults, Ages 60-94. J Aging Phys Act 1999; 7:162-81.

11. Hollenberg M, Yang J, Haight TJ, Tager IB. Longitudinal changes in aerobic capacity implications for concepts of aging. J Gerontol A Biol Sci Med Sci 2006; 61(8):851-8

12. Weiss EP, Spina RJ, Holloszy JO, Ehsani AA. Gender differences in the decline in aerobic capacity and its physiological determinants during the lates decades of life. J Appl Phyiol 2006; 101(3):938-944.

13. Zago AS, Gobbi S. Valores normativos da aptidão funcional de mulheres de 60 a 70 anos. R Bras Ci e Mov 2003; 11(2):77-86.

14. Sorkin JD, Muller DC, Andrés R. Longitudinal change in height of men and women: implictions for interpretation of the body mass index. Am J Epidemiol 1999; 150(9):969-977.

15. World Health Organization. Obesity: preventing and managing the global epidemic. Report of a WHO consultation on obesity. Geneva; 1998.

16. Cabrera MAS, Jacob Filho W. Obesidade em idosos: prevalência, distribuição e associação com hábitos e co-morbidades. Arq Bras Endocrinol Metabol 2001; 45(5):494-501.

17. Santos DM, Sichieri R. Índice de massa corporal e indicadores antropométricos de adiposidade em idosos. Rev Saude Pública 2005; 39(2):163-168.

18. Tavares EL, Anjos LA. Perfil antropométrico da população idosa brasileira: Resultados da pesquisa nacional de saúde e nutrição. Cad Saude Pública 1999; 15(4):759-68.

19. Feijão AMM, Gadelha FV, Bezerro AA, Oliveira AM, Silva MSS, Lima JWO. Prevalência de excesso de peso e hipertensão arterial, em população urbana de baixa renda. Arq Bras Cardiol 2005; 84(1):29-33.

20. Coitinho DC, Leão MM, Recine E, Sichieri R. Condições nutricionais da população brasileira: adultos e idosos. Pesquisa nacional sobre saúde e nutrição. Brasília: INAN, Instituto Nacional de Alimentação e Nutrição; 1991.

21. Gigante DP, Barros FC, Post CLA, Olinto MTA. Prevalência de obesidade em adultos e seus fatores de risco. Rev Saude Publica 1997; 31:236-46.

22. Fundação Instituto Brasileiro de Geografia e Estatística (FIBGE). Síntese de Indicadores Sociais. Uma análise das condições de vida da população Brasileira. Rio de Janeiro: IBGE; 2007. 